\title{
Optimización de la formulación de chocolate oscuro a partir de la mezcla de granos de cacao y contenido de cacao aplicando método de superficie de respuesta
}

\author{
(Optimization of the dark chocolate formulation \\ from the mixture of cocoa beans and cocoa content \\ by applying surface response method)
}

Chire-Fajardo $G^{1}$, Ureña-Peralta $M^{1}$, García-Torres $S M^{1}$, Hartel RW²

\section{Resumen}

En esta investigación se buscó optimizar la formulación de chocolate oscuro a partir de la mezcla de granos de cacao peruano y el contenido de cacao en ella, teniendo como criterio de optimización la mejora de las propiedades físicas del chocolate: color, tamaño de partícula, propiedades reológicas y textura, aplicando la metodología de superficie de respuesta. Se encontró que la formulación óptima presentó una mezcla de granos de cacao de 10 partes de CCN 51 más 90 partes de ICS 6, chocolate oscuro al 70 \% de contenido de cacao, $29.6 \%$ de azúcar y $0.4 \%$ de lecitina. Finalmente, la validación de las propiedades físicas alcanzó el 91.6 \% como promedio.

\section{Palabras clave}

Dureza; formulación de chocolate; maximización; luminosidad; propiedades físicas de chocolate; Perú; propiedades reológicas de chocolate; superficie de respuesta.

\begin{abstract}
This paper sought to optimize the mix of Peruvian cocoa beans and the cocoa content for the dark chocolate formulation, having as an optimization criterion the improvement of the physical properties of chocolate: color, particle size, rheological properties and texture, applying the response surface methodology. It was found that the optimal formulation presented a mixture of COCoa beans of 10 parts of CCN 51 plus 90 parts of ICS 6, dark chocolate at $70 \%$ cocoa content, $29.6 \%$ sugar and $0.4 \%$ lecithin. Finally, the validation of physical properties reached $91.6 \%$ on average.
\end{abstract}

\section{Keywords}

Brightness; formulation of chocolate; chocolate physical properties; chocolate rheological properties; hardness; maximization; Peru; surface response.

\section{Introducción}

Los granos de cacao son comercializados desde los países productores (África, Asia y Sudamérica) a los procesadores de chocolate, alli entran en un proceso de evaluación de su calidad física, química y sensorial realizando el control de calidad de rutina. Algunas empresas que comercializan chocolate en el mundo, usando la pericia de su jurado sensorial, obtienen una mezcla de granos de cacao correspondiente al sabor del chocolate que los identifica, mezcla especial que define las adquisiciones de los granos de cacao de distintos países; sin embargo, no todas las empresas realizan tal práctica y no todos los países productores conocen la mejor mezcla de sus granos de cacao para incrementar sus exportaciones a mejor precio.

\footnotetext{
$1 \quad$ Universidad Nacional Agraria La Molina, Lima, Perú (gchire@lamolina.edu.pe). Universidad Nacional Agraria La Molina, Lima, Perú (moup@lamolina.edu.pe). Universidad Nacional Agraria La Molina, Lima, Perú (mgarcia@lamolina.edu.pe). 
En el Peru, la producción anual de Cacao (Theobroma cacao L.) ha venido creciendo de forma acelerada en los últimos años, en el 2002 la producción anual fue 24353 t cantidad que para el año 2017 incrementó a 120058 t (INEI, 2018). La calidad de sus granos, los cultivares de granos de cacao según los diferentes pisos ecológicos y la biodiversidad biológica en la que se cultivan, tales como: CCN 51 e ICS 6, pueden tener algún componente diferente, así como la creciente comercialización interna y de exportación, hace prever un potencial mercado creciente, generándose la oportunidad de incrementar la exportación no tradicional con valor agregado, ya sea como chocolate o pasta de cacao base para generación de otros productos.

La industria chocolatera en el Perú se viene desarrollando de forma dinámica e innovadora (Vásquez, 2009), mediante una estrategia de promoción genérica de chocolates de calidad. Si un chocolate es muy barato seguro tiene poco o nada de cacao, bajo este criterio se observa qué productos de producción masiva no cumplen con lo normado (35 \% como mínimo de cacao) para ser denominado chocolate (INACAL, 2017). Las dimensiones de la calidad alimentaria depende de atributos intrínsecos (características físico-químicas, composición nutricional, características sensoriales, seguridad microbiológica y toxicológica). La evaluación de estos atributos conlleva la selección de los parámetros a analizar y los métodos más adecuados para la realización de dicho análisis (Torres, 2012). La calidad comercial internacional queda entonces claramente definida por tales parámetros, así como la preferencia del consumidor, cada día más exigente.

En el Perú aún no se ha elaborado un chocolate oscuro de alta calidad para el mercado internacional, utilizando una mezcla de granos de cacao del país que aporten mejores propiedades físicas, quedando aún por definir la composición de mezcla de granos de cacao peruano de alta calidad comercial. Tampoco se tiene una metodología nacional que lleve a obtener mezclas de granos de cacao con calidad de exportación y beneficios para la salud humana.

La optimización de factores en la producción debido a mezclas de granos de cacao y formulaciones en chocolates es una herramienta que permitirá obtener variables de respuestas maximizadas (Aidoo, Afoakwa y Dewettink, 2014) o minimizadas, por lo que se presenta como una metodología adecuada para obtener chocolates con propiedades físicas de calidad requeridas en el proceso.

La presente investigación plantea obtener la formulación óptima de chocolate a partir de la mejora de sus propiedades físicas, aplicando el método de superficie de respuesta, con los factores: mezcla de granos y contenido de cacao.

\section{Metodología}

\subsection{Métodos de análisis}

2.1.1. Análisis físicos: Los chocolates se evaluaron en sus propiedades (Laboratorio de Ciencia de Alimentos, University of Wisconsin-Madison, USA):

Pasta de chocolate. Después del concado el chocolate fue evaluado en sus propiedades reológicas: viscosidad y esfuerzo mínimo de fluencia (Minifie, 1999), fueron medidos en el Reómetro hibrido Discovery HR-2 (TA Instruments, New Castle, DE, U.S.A.), equipado con plomada y copa. La masa de chocolate fue derretida a $50{ }^{\circ} \mathrm{C}$ y mantenida por 60 minutos, asegurando la destrucción del historial del cristal de grasa. Luego el chocolate fue mezclado para homogeneizar y colectar la muestra. La muestra de chocolate líquida $(25 \mathrm{ml}$, equivalente a $30 \mathrm{~g}$ aproximadamente), fue incorporada a la copa a la temperatura de $40{ }^{\circ} \mathrm{C}$. Bajando la plomada en funcionamiento en el espacio operativo $\left(5917 \mathrm{~nm}\right.$ ) la muestra fue mantenida a $40^{\circ} \mathrm{C}$ por 5 minutos 
para alcanzar el equilibrio térmico, seguidamente la muestra fue pre-cizallada a una tasa de corte constante de $76 \mathrm{~s}^{-1}$ a $40{ }^{\circ} \mathrm{C}$ por otros 5 minutos. El barrido de flujo fue realizado a un paso logarítmico, a una tasa de corte de 1000 a $1 \mathrm{~s}^{-1}$ y a una temperatura constante de $40{ }^{\circ} \mathrm{C}$. Los datos fueron procesados en el software del reómetro (TRIOS) y se ajustó al modelo de Casson (Wolf, 2017; Nelson y Beckett, 1994) mostrada en la ecuación (1). Después de cada medida, se limpiaron la plomada y la copa. Se evaluaron tres repeticiones por unidad experimental.

$$
(1+a) \sqrt{D_{N}}=\frac{1}{\sqrt{C A}}(1+a) \sqrt{1} 2 \sqrt{C A}
$$

Dónde: $\mathrm{a}$ = relación del radio interior al exterior, $\mathrm{D}_{\mathrm{N}}=$ régimen de cizalladura aplicando $\left(\mathrm{D}_{\mathrm{N}}>1\right)$ al cilindro externo $\left(\mathrm{s}^{-1}\right), \mathrm{h}_{\mathrm{CA}}=$ viscosidad, $\tau_{1}=$ fuerza de cizalladura transmitida a la superficie del cilindro interior (dinas $\mathrm{cm}^{-2}$ ), $\tau_{\mathrm{CA}}=$ esfuerzo mínimo de Casson (dinas $\mathrm{cm}^{-2}$ ).

Tableta de chocolate. Después del almacenamiento a $20 \pm 2{ }^{\circ} \mathrm{C}$ por 14 días (Afoakwa, Paterson, Fowler \& Vieira, 2008) en la tableta de chocolate, se evaluaron las propiedades físicas:

- Color: el color de las muestras de chocolate fue medida con el colorímetro Minolta CR-300 (Minolta Corporation, Ramsey, New Jersey), utilizando un sistema de escala de color CIELAB con medidas de $L^{*}$. El valor $L^{*}$ mide la escala de oscuridad a claridad. Todas las medidas fueron realizada a la luz del día. Se evaluaron cinco repeticiones por unidad experimental (tomadas en posición de cuatro ángulos 0, 90, 180, y 270 , Chire, Valdivia, Orihuela \& Ureña, 2017).

- Textura: las muestras de chocolate fueron evaluadas en un analizador de textura TA.TX plus (England) con software EXPONENT y una geometría de aguja P:2 (Texture Technologies) (Afoakwa, et al. 2008). La dureza fue reportada como una fuerza de penetración máxima $(\mathrm{N})$ requerida para que la aguja penetre la muestra de chocolate (diámetro $50 \mathrm{~mm}$ y grosor $5 \mathrm{~mm}$ ) sobre una distancia de $5 \mathrm{~mm}$ a una velocidad constante de $2 \mathrm{~mm} / \mathrm{s}$. Se evaluaron tres repeticiones por unidad experimental.

- Tamaño de partícula: fue determinado por el método del micrómetro MITUTOYO® que solo detecta partículas grandes (Beckett, 2008). Se limpiaron las superficies planas de las mandíbulas del micrómetro y se ajustó a cero. Se mezcló en un recipiente 50 partes de aceite de girasol con 50 partes de chocolate fundido, se colocó una gota de la emulsión en una de las mandíbulas del micrómetro, se enroscó las mandíbulas hasta que fácilmente se midieron las partículas del producto y se tomó la lectura en micras. Se abrió las mandíbulas y se limpiaron para la siguiente medida. Se evaluaron tres repeticiones por unidad experimental.

2.1.2. Análisis estadístico. Todos los datos se expresaron como el promedio con su desviación estándar. El análisis estadístico se realizó con el Programa Statgraphics ® Plus mediante un ANOVA, para los factores (mezcla de granos y contenido de cacao en la formulación de chocolate) con un diseño completo al azar (DCA). 


\subsection{Metodología experimental}

\subsubsection{Obtención de chocolate oscuro a partir de formulaciones en base a mezclas de cacao CCN 51 e $1 C S 6$}

Los granos de cacao fermentados fueron adquiridos del Fundo Terra Nostra (Uchiza, Perú) y almacenados a temperatura de $20 \pm 1^{\circ} \mathrm{C}$ y humedad relativa (HR) de 50-60 \% antes de su uso (Laboratorio de Análisis Fisicoquímico de Alimentos, UNALM, Lima, Perú). Se trabajaron con dos varietales de granos de cacao: CCN 51 (Colección Castro Naranjal 51) por el mayor volumen de producción en el país e ICS 6 (Imperial Collage Selection 6) (García, 2014) por su calidad en fino aroma (Cultivar trinitario). La mezcla de estos granos de cacao se realizó a tres niveles: 10:90, 50:50 y 90:10.

Se realizó el análisis proximal de cada cultivar de grano de cacao (AOAC, 2016) (Laboratorio de Fisicoquímica de Alimentos, UNALM, Lima, Perú). Luego se procesaron los tres niveles de mezclas en lotes de $13 \mathrm{~kg}$ cada uno, con dos repeticiones (Chocomuseo, Lima, Perú), los mismos que pasaron por las operaciones unitarias estándar de procesamiento industrial (Gutiérrez, 2017) para la obtención de derivados del cacao, las que fueron: "tostado", "descascarillado", "molienda" y "refinado". Las pastas, como primer derivado del cacao, fueron prensadas para la obtención de la manteca (segundo derivado) y torta de cacao (tercer derivado) (Planta de procesamiento de cacao "Warmitech", Saposoa, Perú). La torta de cacao fue molida hasta la obtención de cocoa en polvo (cuarto derivado). La pasta, manteca y cocoa, constituyeron parte de los ingredientes requeridos en la formulación del chocolate oscuro.

Se determinaron los contenidos de grasa de la pasta de cacao y cocoa en polvo (AOAC 920.75a: Método Soxhlet) (AOAC, 2016) (Laboratorio de Análisis fisicoquímico de Alimentos, Lima, Perú), para realizar el balance de contenido de grasa y sólidos no grasos en la formulación de chocolate oscuro.

Se formularon los diferentes tipos de chocolate oscuro según porcentaje de cacao (60, 70 \% y $80 \%$ \%) (INACAL, 2017; Wohlmuth, 2017). Posteriormente, lotes de $1200 \mathrm{~g}$ de chocolate oscuro fueron procesados (Fábrica de chocolates Cacao Valley, Lima, Perú). El contenido de cacao corresponde a la suma de los ingredientes del cacao como: pasta de cacao, cocoa en polvo y manteca de cacao. En la formulación los ingredientes fijos fueron: pasta de cacao, manteca de cacao y lecitina de soya, los demás ingredientes fueron variando según porcentaje de cacao por tipo de producto, es decir a un mayor contenido de cacao, se reducía el azúcar para incrementar el contenido de cocoa en polvo, así en forma escalonada se llegó a tener el porcentaje de cacao en cada tipo de chocolate. Las formulaciones de $70 \%$ y $80 \%$ de cacao tuvieron como ingredientes: pasta y manteca de cacao, cocoa en polvo, azúcar blanca refinada y lecitina de soya, que fueron adquiridos de un mismo lote. La formulación de 60 \% de cacao no llevó cocoa en polvo.

La elaboración del chocolate se realizó en condiciones similares para las distintas formulaciones ensayadas (Gutiérrez, 2017), siendo sus etapas: el mezclado de ingredientes, donde se adicionó la lecitina de soya; el refinado de 12 horas, el concado de 12 horas; el envasado en contenedores de plástico con tapa; y el almacenado a temperatura de $19-20{ }^{\circ} \mathrm{C}$ y HR de $50-60 \%$. Posteriormente, la masa de chocolate fue atemperada por un sistema termocíclico o ciclotérmico definido para cada tipo de formulación (Kleinert, 1980; Miquel, Carli, Couzend, Wille y Hall, 2001; Windhab, 2017) (Laboratorio de Ciencia de Alimentos, University of Wisconsin-Madison, USA).

En el atemperado el chocolate fue derretido en el rango de $45-55^{\circ} \mathrm{C}$, luego, sucesivamente, fue enfriado, calentado y enfriado. Una vez que el chocolate llegó a la temperatura final de 30-30.5 ${ }^{\circ} \mathrm{C}$, adquiriendo una consistencia viscosa, fue moldeado en piezas de superficie plana, 
y enfriados posteriormente de $19-20{ }^{\circ} \mathrm{C}$ a una humedad relativa de 50\%-60 \% hasta su cristalización. Seguidamente, se desmoldaron los chocolates y sin empacar se almacenaron en un ambiente a $19-20^{\circ} \mathrm{C}$ y humedad relativa de $50 \%-60 \%$ para la posterior evaluación de sus propiedades físicas.

\subsubsection{Optimización}

Para la elección de la formulación óptima de chocolate oscuro por sus mejores propiedades físicas, se utilizó el método de optimización por superficie de respuesta con un factorial $3^{2}$, siendo los factores y sus niveles: la mezcla de granos (10:90, 50:50 y 90:10) y el contenido de cacao en la formulación de chocolate (60 \%, 70 \% y 80 \%), obteniéndose un total de nueve tratamientos con dos repeticiones. Se utilizó la ecuación de segundo orden (2).

$$
Y=\beta_{b}+\sum_{i=1}^{k} \beta_{i} X_{i}+\sum_{i=1}^{k} \beta_{i i} X_{i}^{2}+\sum_{i=1}^{k-1} \sum_{j=2}^{k} \beta_{i j} X_{i} X_{j}+\varepsilon
$$

La optimización de los parámetros se realizó mediante métodos gráficos, de acuerdo a lo obtenido con los modelos de regresión, y utilizando la deseabilidad máxima del sistema de múltiples respuestas. Esta es una función cuyo rango de valores varía en el intervalo de $(0,-1)$ y resulta de promediar las medias geométricas de las deseabilidades individuales de cada variable de respuesta, como se muestra en la ecuación (3).

$$
D(n)=(d 1, d 2, \ldots \ldots ., d n) 1 / n
$$

Donde $d 1, d 2, \ldots ., d n$ son las respuestas y $n$ es el número total de respuestas en la medición. El valor más alto de $D(n)$ indica la mejor combinación del sistema. Para las variables de respuestas: viscosidad y esfuerzo mínimo de fluencia Casson, color $\left(L^{*}\right)$ y tamaño de partícula, se buscó la minimización; mientras que, para la dureza se buscó la maximización.

Todas las variables fueron introducidas en el programa Statgraphics ${ }^{\oplus}$. Se determinaron las ecuaciones ajustadas de cada propiedad física: viscosidad de Casson $\left(\mathrm{h}_{\mathrm{CA}}\right)$ esfuerzo mínimo de Casson $\left(\tau_{\mathrm{CA}}\right)$ color $\left(\mathrm{L}^{*}\right)$, dureza (D) y tamaño de partícula (TP), en función a los factores: mezcla de granos de cacao (M) y contenido de cacao en la formulación (C), también se reportó el coeficiente de regresión $\left(R^{2}\right)$ y error estándar de la estima (EEE). Se determinó el efecto de las propiedades físicas en función de los factores y se conoció el nivel óptimo de cada factor.

\subsubsection{Elección de la formulación óptima del chocolate oscuro}

Con los valores de optimización obtenidos, se definieron los niveles de los factores: proporción de mezcla de granos de cacao y contenido de cacao en la formulación. Se utilizó el programa estadístico Statgraphics ® Plus para los cálculos correspondientes.

2.2.4 Validación de la formulación óptima de chocolate oscuro a partir de sus propiedades físicas: Considerando los niveles de los factores que definen la formulación óptima se realizó el procesamiento del chocolate con tres repeticiones (Fábrica de chocolates, Cacao Valley, Lima, Perú), y posteriormente se analizaron las propiedades físicas, con el fin de comparar los valores estimados por el modelo y los valores observados proveniente de las mediciones de las propiedades físicas de la formulación óptima. Se empleó la ecuación de validación (García, 2018). 


\section{Resultados y discusión}

\subsection{Obtención de chocolate oscuro a partir de formulaciones con base en mezclas de cacao CCN 51 e ICS 6}

Los granos de cacao CCN 51 e ICS 6 presentaron valores diferentes en el análisis proximal (Tabla 1). En el estudio de Tonfack et al. (2018) para cacao Trinitario de Camerún se reporta humedad $(5.9 \pm 0.6 \%)$, grasa cruda $(41.2 \pm 0.8 \%)$, carbohidratos totales $(27.7 \pm 0.1 \%)$, proteína cruda $(17.9 \pm 0.3 \%)$ y ceniza $(7.3 \pm 0.4 \%)$, que son valores diferentes a los encontrados en la presente investigación. Adeyeye et al. (2010) reportan para cacao de Nigeria proteína cruda (13.6 \pm 0.30 $\%$ en cacao no fermentado y $15.2 \pm 0.21 \%$ en cacao fermentado), valores superiores encontradas para los varietales de cacao fermentado de la presente investigación.

Tabla 1. Composición proximal de granos de cacao fermentado

\begin{tabular}{|l|l|l|}
\hline \multicolumn{1}{|c|}{ Componentes (\%) } & \multicolumn{1}{|c|}{ CCN 51 } & \multicolumn{1}{c|}{ ICS 6 } \\
\hline Humedad & $6.72 \pm 0.05^{\mathrm{a}}$ & $6.76 \pm 0.07 \mathrm{~b}$ \\
\hline Grasa cruda & $40.67 \pm 0.11^{\mathrm{a}}$ & $42.73 \pm 0.05 \mathrm{~b}$ \\
\hline Proteína cruda & $13.66 \pm 0.25 \mathrm{~b}$ & $12.56 \pm 0.10 \mathrm{a}$ \\
\hline Ceniza & $3.02 \pm 0.01^{\mathrm{a}}$ & $3.27 \pm 0.01 \mathrm{~b}$ \\
\hline Fibra cruda & $5.40 \pm 0.03 \mathrm{~b}$ & $4.51 \pm 0.01 \mathrm{a}$ \\
\hline Carbohidratos totales & $35.94 \pm 0.36 \mathrm{~b}$ & $34.68 \pm 0.13^{\mathrm{a}}$ \\
\hline Total & 100.00 & 100.00 \\
\hline
\end{tabular}

Los resultados se expresan como promedio \pm desviación estándar de tres repeticiones. Las distintas letras en la misma fila indican diferencias significativas $(p \leq 0.05)$ de menor a mayor en orden alfabético.

Como resultado del procesamiento de los granos de cacao se obtuvo como primer derivado las pastas correspondientes a cada nivel de mezcla ensayado. El contenido graso promedio de estas fue $54.84 \pm 1.80 \%$. La cocoa, como cuarto derivado del cacao, tuvo un contenido graso promedio de $22.67 \pm 1.74 \%$.

Con los contenidos grasos promedio de las pastas y las cocoas, se formularon los chocolates oscuros con $60 \%, 70 \%$ y $80 \%$ de cacao, considerando no superar el valor de $40 \%$ de grasa, obteniéndose 33.8; 36.0 y $38.3 \%$, respectivamente (Tabla 2). El valor máximo de $40 \%$ de grasa fue definido considerando aspectos nutricionales (9 kcal/g de grasa) y tecnológicos (dificultad operativa en el atemperado y moldeado).

Tabla 2. Formulaciones del chocolate oscuro en base al contenido de cacao

\begin{tabular}{|l|c|c|c|}
\hline \multicolumn{1}{|c|}{ Ingredientes } & $\mathbf{6 0} \mathbf{~ g ~ c a c a o} / \mathbf{1 0 0} \mathbf{~ g}$ & $\mathbf{7 0} \mathbf{~ g ~ c a c a o} / \mathbf{1 0 0} \mathbf{~ g}$ & $\mathbf{8 0 ~} \mathbf{~ g ~ c a c a o / 1 0 0 ~} \mathbf{~}$ \\
\hline Pasta de cacao & 58.0 & 58.0 & 58.0 \\
\hline Azúcar blanca refinada & 39.6 & 29.6 & 19.6 \\
\hline Cocoa en polvo & 0.0 & 10.0 & 20.0 \\
\hline Manteca de cacao & 2.0 & 2.0 & 2.0 \\
\hline Lecitina de soya & 0.4 & 0.4 & 0.4 \\
\hline Total & 100.0 & 100.0 & 100.0 \\
\hline \% Grasa & 33.8 & 36.0 & 38.3 \\
\hline \% Solidos no grasos & 26.2 & 34.0 & 41.7 \\
\hline \% Cacao & 60.0 & 70.0 & 80.0 \\
\hline
\end{tabular}




\subsection{Determinación de la fórmula óptima del chocolate oscuro}

3.2.1 Pasta de chocolate: al término del proceso de concado se midieron las propiedades reológicas del chocolate: viscosidad y esfuerzo mínimo de fluencia según el modelo de Casson (Minifie, 1999) (Tabla 3).

Tabla 3. Valores de propiedades reológicas

del chocolate oscuro según modelo Casson

\begin{tabular}{|l|l|l|l|}
\hline \multicolumn{1}{|c|}{$\begin{array}{c}\text { Mezcla } \\
\text { CCN 51: ICS 6 }\end{array}$} & \multicolumn{1}{|c|}{$\begin{array}{c}\text { Cacao } \\
(\%)\end{array}$} & Viscosidad de Casson (Pa.s) $\mathbf{h}_{\mathrm{CA}}$ & \multicolumn{1}{|c|}{$\begin{array}{c}\text { Esfuerzo mínimo } \\
\text { de fluencia de Casson } \\
(\mathrm{Pa}) \tau_{\mathrm{CA}}\end{array}$} \\
\hline $50: 50$ & 70 & $2.95 \pm 0.04$ & $18.42 \pm 0.49$ \\
\hline $90: 10$ & 70 & $2.82 \pm 0.20$ & $15.11 \pm 1.12$ \\
\hline $50: 50$ & 80 & $3.23 \pm 0.04$ & $21.19 \pm 0.74$ \\
\hline $10: 90$ & 80 & $2.86 \pm 0.33$ & $25.19 \pm 2.32$ \\
\hline $90: 10$ & 60 & $2.59 \pm 0.09$ & $15.09 \pm 1.26$ \\
\hline $90: 10$ & 80 & $3.57 \pm 0.42$ & $22.16 \pm 0.41$ \\
\hline $10: 90$ & 70 & $2.52 \pm 0.04$ & $12.32 \pm 0.41$ \\
\hline $10: 90$ & 60 & $2.35 \pm 0.05$ & $8.99 \pm 1.11$ \\
\hline $50: 50$ & 60 & $2.20 \pm 0.03$ & $8.45 \pm 0.22$ \\
\hline
\end{tabular}

En la elaboración del chocolate se debe controlar la viscosidad y el esfuerzo mínimo de fluencia según modelo de Casson, ya que la pasta a $40{ }^{\circ} \mathrm{C}$ se almacena en tanques, pasando por tuberías, bombas y equipos, lo que requiere de una fluencia que permita su desplazamiento y evitar con ello la acumulación de sólidos en las paredes, así como la generación de burbujas de aire dentro de la masa del chocolate. En el momento final de la operación unitaria de "atemperado", la pasta de chocolate se encuentra a temperatura de $32^{\circ} \mathrm{C}$, lo que obliga a un mayor control de la fluencia para evitar la cristalización temprana y bajos rendimientos en producción durante el "moldeado".

Según lo expuesto en la Tabla 3, la mezcla 50 CCN 51:50 ICS 6 para 60 \% de cacao, es la que presentó el menor valor de viscosidad (2.20 \pm 0.03 Pa.s), entre todas las demás mezclas y cantidades de cacao ensayadas, la ecuación de regresión (Tabla 4) donde los factores con efectos significativos fueron (Tabla 5): Mezcla y Contenido de cacao ( $p \leq 0.05$ ). En el estudio de Aidoo et al. (2014) trabajaron chocolate oscuro al $51.6 \%$ de cacao y obtuvieron valores de viscosidad de Casson de $2.40 \pm 0.09$ Pa.s y $2.40 \pm 0.05$ Pa.s, que son superiores a los resultados en esta investigación para 60 \% de contenido de cacao. Para el esfuerzo mínimo de fluencia (Tabla 3), la mezcla 50 CCN 51:50 ICS 6 para $60 \%$ de cacao presentó el menor valor (8.45 \pm 0.22 Pa), la ecuación de regresión (Tabla 4), donde los factores con efectos significativos fueron (Tabla 5): Contenido de cacao y la interacción Mezcla/Contenido de cacao ( $\leq \leq 0.05)$. 
Tabla 4. Ecuaciones de optimización para las propiedades físicas de pasta y tableta de chocolate

\begin{tabular}{|c|c|c|c|}
\hline Propiedad física & Ecuación de regresión & $\mathbf{R}^{2}$ & EEE \\
\hline Viscosidad de Casson $\left(\mathrm{h}_{\mathrm{CA}}\right)$ & $\begin{array}{l}h_{C A}=2.40-0.015^{*} M-0.024^{*} C-0.0000068 * M^{2}+0.00029 * M * C \\
+0.00037^{*} C^{2}\end{array}$ & 83.89 & 0.22 \\
\hline $\begin{array}{l}\text { Esfuerzo mínimo de fluen- } \\
\text { cia de Casson }\left(\tau_{C A}\right)\end{array}$ & $\begin{array}{l}\tau_{\mathrm{CA}}=28.89+0.400 * M-1.297^{*} C+0.0002822^{*} M^{2}-0.00570^{*} M^{*} C \\
+0.01559^{*} C^{2}\end{array}$ & 88.31 & 2.44 \\
\hline Color $\left(L^{*}\right)$ & $\begin{array}{l}L^{*}=27.87+0.027^{*} M-0.032^{*} C+0.0000089^{*} M^{2}-0.00031^{*} M^{*} C \\
+0.00014^{*} C^{2}\end{array}$ & 83.34 & 0.18 \\
\hline Dureza (D) & $\begin{array}{l}D=-127.01-0.175^{\star} M+4.549^{*} C-0.0013068^{*} M^{2}+0.00415^{\star} M{ }^{*} C \\
-0.03081^{\star} C^{2}\end{array}$ & 81.64 & 2.55 \\
\hline Tamaño de partícula (TP) & $\begin{array}{l}T P=22.82-0.132 * M+0.272^{*} C-0.000427^{*} M^{2}+0.00275^{*} M{ }^{*} C \\
-0.00358^{*} C^{2}\end{array}$ & 27.09 & 2.66 \\
\hline
\end{tabular}

Leyenda:

$\mathrm{h}_{\mathrm{CA}}=$ Viscosidad de Casson

$\tau_{\mathrm{CA}}=$ Esfuerzo mínimo de fluencia de Casson

$L^{*}=$ Color

$\mathrm{D}=$ Dureza

TP = Tamaño de partícula

$M=$ Mezcla de granos de cacao

$C=$ Contenido de cacao en la formulación

$\mathrm{R}^{2}=$ Coeficiente de regresión

$\mathrm{EEE}=$ Error estándar de la estima

Tabla 5. Efecto de las propiedades físicas en la optimización

de la formulación del chocolate oscuro en pasta y tableta

\begin{tabular}{|l|l|l|}
\hline \multicolumn{1}{|c|}{ Propiedad física } & \multicolumn{1}{|c|}{ Factores } & \multicolumn{1}{c|}{ Significancia } \\
\hline Viscosidad de Casson $\left(h_{C A}\right)$ & $\begin{array}{l}\text { Mezcla } \\
\text { Contenido de cacao }\end{array}$ & $*$ \\
\hline Esfuerzo mínimo de fluencia de Casson $\left(\tau_{C A}\right)$ & $\begin{array}{l}\text { Contenido de cacao } \\
\text { Interacción Mezcla/Contenido de cacao }\end{array}$ & $*$ \\
\hline Color $\left(L^{*}\right)$ & $\begin{array}{l}\text { Mezcla } \\
\text { Contenido de cacao }\end{array}$ & $*$ \\
\hline Dureza $(D)$ & Contenido de cacao & $*$ \\
\hline Tamaño de partícula $(T P)$ & Ninguno & N.S. \\
\hline
\end{tabular}

Leyenda:

$\mathrm{h}_{\mathrm{CA}}=$ Viscosidad de Casson

$\tau_{\mathrm{CA}}=$ Esfuerzo mínimo de fluencia de Casson

$L^{*}=$ Color

$\mathrm{D}=$ Dureza

TP = Tamaño de partícula 
3.2.2 Tableta de chocolate: Al término del almacenamiento, se midieron las propiedades físicas de las tabletas de chocolate: color ( $\left.\mathrm{L}^{*}\right)$, dureza (N) y tamaño de partícula (micras). Los resultados se muestran en la Tabla 6.

Tabla 6. Valores observados de propiedades físicas del chocolate oscuro

\begin{tabular}{|l|c|c|c|c|}
\hline $\begin{array}{c}\text { Mezcla } \\
\text { CCN 51: ICS 6 }\end{array}$ & $\begin{array}{c}\text { Contenido } \\
\text { de cacao }\end{array}$ & $\begin{array}{c}\text { Color } \\
\left(\mathbf{L}^{*}\right)\end{array}$ & $\begin{array}{c}\text { Dureza } \\
(\mathbf{N})\end{array}$ & $\begin{array}{c}\text { Tamaño } \\
\text { de partícula } \\
\text { (micras) }\end{array}$ \\
\hline $50: 50$ & 70 & $26.60 \pm 0.39$ & $44.20 \pm 0.35$ & $23.8 \pm 2.1$ \\
\hline $90: 10$ & 70 & $26.77 \pm 0.18$ & $41.04 \pm 1.74$ & $25.7 \pm 0.0$ \\
\hline $50: 50$ & 80 & $26.30 \pm 0.05$ & $45.63 \pm 0.70$ & $27.8 \pm 2.1$ \\
\hline $10: 90$ & 80 & $26.19 \pm 0.34$ & $41.19 \pm 0.00$ & $20.2 \pm 2.6$ \\
\hline $90: 10$ & 60 & $27.28 \pm 0.30$ & $31.63 \pm 0.07$ & $26.8 \pm 0.7$ \\
\hline $90: 10$ & 80 & $26.46 \pm 0.01$ & $41.80 \pm 0.32$ & $25.5 \pm 0.7$ \\
\hline $10: 90$ & 70 & $26.39 \pm 0.05$ & $39.35 \pm 0.68$ & $27.8 \pm 0.7$ \\
\hline $10: 90$ & 60 & $26.50 \pm 0.19$ & $37.66 \pm 4.17$ & $25.8 \pm 0.2$ \\
\hline $50: 50$ & 60 & $26.86 \pm 0.14$ & $32.67 \pm 2.60$ & $26.3 \pm 0.5$ \\
\hline
\end{tabular}

El color marrón del chocolate oscuro, correspondiente a valores bajos de $L^{*}$, se logra por varios factores: el color de grano, la temperatura y los tiempos de tostado y concado, la formulación del chocolate, el buen atemperado, entre otros. Según lo expuesto en la Tabla 6, la mezcla 10 CCN 51:90 ICS 6 para $80 \%$ de cacao obtuvo el menor valor de L* (26.19 \pm 0.34$)$, la ecuación de regresión (Tabla 4), donde los factores con efectos significativos fueron (Tabla 5): Mezcla y Contenido de cacao ( $p \leq 0.05)$.

La dureza en la industria chocolatera para barras o tabletas, que sensorialmente está asociada con el snap o sonido al fracturarse el producto (Wohlmuth, 2017), y que en términos físicos está asociado a la fuerza de ruptura, es resultado de la formulación, atemperado y enfriamiento, siendo mayor en chocolates oscuros que en los que contienen leche y, en Perú, los oscuros importados en comparación de los oscuros nacionales (Chire et al. 2017). Según lo expuesto en la Tabla 6, la mezcla 50 CCN 51:50 ICS 6 para 80 \% de cacao presentó el mayor valor de dureza (45.63 $\pm 0.7 \mathrm{~N}$ ), la ecuación de regresión (Tabla 4), donde el factor con efecto significativo (Tabla 5) fue el Contenido de cacao ( $\mathrm{p} \leq 0.05$ ).

El tamaño de partícula se logra en el refinado y, en menor medida, en el concado. Se busca que su valor sea el menor posible para que el chocolate se disuelva fácilmente con la temperatura bucal. Según lo expuesto en la Tabla 6, la mezcla 10 CCN 51:90 ICS 6 para 80 \% de cacao presentó el menor valor de tamaño de partícula (20.2 \pm 2.6 micras), la ecuación de regresión (Tabla 4), donde no hubo factores (Tabla 5) con efectos significativos ( $p>0.05$ ), es decir el tamaño de partícula no depende de los factores en estudio, con lo que el menor valor de tamaño de partícula se podría alcanzar también con los niveles mínimos de cada factor.

Para determinar la formulación óptima del chocolate oscuro a partir de las propiedades físicas evaluadas, se aplicó el procedimiento estadístico de optimización de múltiples respuestas del software Statgraphics $₫$ plus, teniendo como resultado una superficie estimada (Figura 1) valores estimados (Tabla 7), donde su ecuación está definida por el grado de deseabilidad (variables 
respuestas: viscosidad y esfuerzo mínimo de Casson, color $\left(L^{*}\right)$, dureza y tamaño de partícula) en función de los factores Mezcla y Contenido de cacao (porcentaje de cacao). La formulación óptima para 0.64 de deseabilidad fue la correspondiente a la mezcla 10 CCN 51:90 ICS 6, con 69.71 \% de contenido de cacao, según Lazic (2004) citado por Bacio (2007) le correspondería a tal formulación un calificativo de "bueno". Esan, Sobukola, Sanni, Bakare y Muñoz (2015) encontraron condiciones óptimas de temperatura y tiempo en fritura, con una deseabilidad máxima de 0.61 valor inferior a la presente investigación. Con fines de validación de los resultados se realizó una producción de chocolate oscuro con los niveles de los dos factores que hacen óptima la formulación, siendo el contenido de cacao, por aproximación al valor entero, de 70 \%.

Figura 1. Superficie de respuesta para el chocolate oscuro

Superficie de Respuesta Estimada

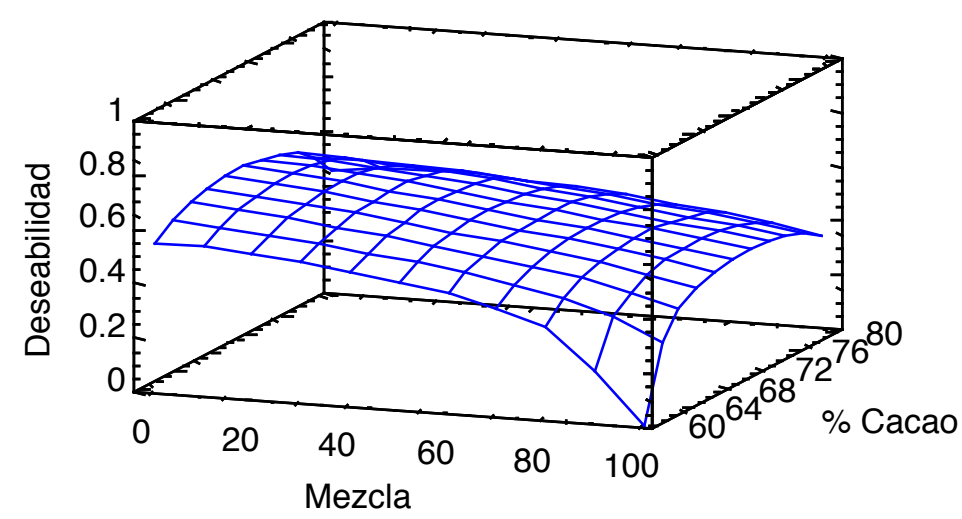

\subsubsection{Caracterización del chocolate oscuro elaborado con la formulación óptima}

Se presenta el valor estimado de cada una de las propiedades físicas del chocolate oscuro elaborado con base en la formulación que resultó óptima, aplicando el método de superficie de respuesta (valor estimado por Statgraphics ${ }^{\circledR}$ ), así como el valor observado obtenido para la validación de la optimización (Tabla 7). La viscosidad de Casson resultó menor a lo observado y se encuentra en el rango a lo reportado por Dwi et al. (2019), quienes obtuvieron valores para distintas formulaciones (pasta de cacao, manteca de cacao, cocoa en polvo), contenidos de cacao (51.6-51.8 \%) y de grasa (33-36 \%). El esfuerzo mínimo de fluencia (14.22 Pa) está dentro del rango obtenido experimentalmente (observados), siendo mayor a lo reportado por Aidoo et al. (2014) y Dwi et al. (2019) para chocolate oscuro con 51.6 \% y 51.6-51.8 \% de cacao respectivamente. El color (26.37 L*) obtenido de la optimización, donde el varietal ICS 6 otorga un color más oscuro a la mezcla que el varietal CCN 51, está dentro del rango de lo observado y es menor a lo reportado por Aidoo et al. (2014) para chocolate con 51.6 \% de cacao.

La dureza es de $42.03 \pm 0.12 \mathrm{~N}$ superior a la dureza del correspondiente estimado (41.37 $\mathrm{N})$ y del valor observado para la misma mezcla de granos $(39.35 \pm 0.68 \mathrm{~N})$, la que está relacionada al contenido de sólidos grasos a $20{ }^{\circ} \mathrm{C}$ con un valor de $73.3 \pm 0.20$ a $76.20 \pm 0.40 \%$ (Tran, Van Durme, Van de Ville, De Winne, Delbaere, De Clercq, Pan, Nguyen, Tran \& Dewettinck, 2016), así como el contenido de fibra de 7 \% y 13 \% (Ostrowska-Ligeza, Marzec, Górska, WirkowskaWojdyła, Brys, Rejch y Czarkowska, 2018) en un chocolate con una dureza en el rango de 124.6 
$\pm 16.5 \mathrm{~N}$ y $177.6 \pm 12.7 \mathrm{~N}$; dureza que determina la vida en anaquel y que está relacionada con la composición del chocolate y la cristalización de la grasa durante el proceso (enfriamiento). Sin embargo, este valor resulta mayor a lo reportado por Aidoo et al. (2014), Chire et al. (2017) y por Alvis, Perez y Arriazola (2011) quienes encontraron para chocolate oscuro valores de dureza de $15.0 \pm 0.7 \mathrm{~N}$ a $38.3 \pm 1.9 \mathrm{~N}$. Por el tamaño de partícula (25.90 \pm 0.70 micras) podría ser utilizado en la elaboración de tabletas con inclusiones (frutas, nueces y cereales), según lo reportado por Wohlmuth (2017), quien clasifica a los chocolates oscuros, en barra o tableta, de alta calidad (15-20 micras), mediana calidad (22-25 micras) y los que llevan inclusiones (25-30 micras).

Tabla 7. Valores de propiedades físicas del chocolate oscuro elaborado con la formulación óptima

\begin{tabular}{|c|c|c|c|c|c|c|}
\hline \multirow{2}{*}{ Propiedad física } & \multicolumn{2}{|c|}{ Valor } & \multirow{2}{*}{$\begin{array}{l}\text { Aidoo et al. } \\
\text { (2014) }\end{array}$} & \multicolumn{2}{|c|}{ Chire et al. (2017) } & \multirow{2}{*}{$\begin{array}{l}\text { Dwi et al. } \\
(2019)\end{array}$} \\
\hline & Estimado & Observado & & Perú* & Otros ${ }^{\star \star}$ & \\
\hline $\begin{array}{l}\text { Viscosidad de } \\
\text { Casson (Pa.s) } h_{C A}\end{array}$ & 2.54 & $3.07 \pm 0.46$ & $\begin{array}{l}2.40 \pm 0.09 \\
2.40 \pm 0.05\end{array}$ & - & - & $\begin{array}{l}1.29 \pm 0.05 \\
3.09 \pm 0.01\end{array}$ \\
\hline $\begin{array}{l}\text { Esfuerzo mínimo } \\
\text { de fluencia de } \\
\text { Casson }(\mathrm{Pa}) \tau_{\mathrm{CA}}\end{array}$ & 14.22 & $12.28 \pm 4.36$ & $\begin{array}{l}10.24 \pm 0.14 \\
10.15 \pm 0.67\end{array}$ & - & - & $\begin{array}{l}3.47 \pm 0.06 \\
13.13 \pm 0.48\end{array}$ \\
\hline Color $\left(L^{\star}\right)$ & 26.37 & $26.53 \pm 0.23$ & $\begin{array}{l}28.40 \pm 0.07 \\
28.68 \pm 0.19\end{array}$ & $\begin{array}{l}29.44 \pm \\
1.06\end{array}$ & $\begin{array}{l}29.80 \quad \pm \\
2.68 \\
\end{array}$ & \\
\hline Dureza (N) & 41.37 & $42.03 \pm 0.12$ & $\begin{array}{l}3.79 \pm 0.12 \\
3.79 \pm 0.17\end{array}$ & $\begin{array}{l}9.01 \\
1.92\end{array}$ & $9.52 \pm 2.72$ & - \\
\hline $\begin{array}{l}\text { Tamaño de partí- } \\
\text { cula (micras) }\end{array}$ & 24.90 & $25.90 \pm 0.70$ & $\begin{array}{l}32.40 \pm 0.27 \\
31.96 \pm 0.90\end{array}$ & $\begin{array}{l}20.00 \pm \\
2.00\end{array}$ & $19.10 \pm 2.90$ & \\
\hline
\end{tabular}

${ }^{*}$ Chocolates oscuros fabricados en Perú.

**Chocolates oscuros importados que se comercializan en el Perú.

Valor estimado = obtenido de la optimización con superficie de respuesta.

Valor observado = obtenido de los ensayos para la validación de la optimización.

\subsubsection{Validación de la formulación óptima}

El grado de validación promedio de la formulación óptima (91.6 \%) a partir de los valores observados de: viscosidad, esfuerzo mínimo de fluencia, color, dureza y tamaño de partícula se muestran en la Tabla 8. Se determinó de los datos reportados por Erdem et al. (2014) un grado de validación de $95.3 \%$ en la optimización del proceso a partir de propiedades físicas y sensoriales para obtener un chocolate simbiótico aplicando superficie de respuesta. En la optimización de la fritura de papas nativas García (2018) obtuvo una validación de 76.7 \% y Talancha (2014) 97.0 \% en la optimización de la incorporación de dos ingredientes en la formulación de mayonesa para maximizar su aceptabilidad. 
Tabla 8. Valores de validación de las propiedades físicas del chocolate oscuro elaborado con la formulación óptima

\begin{tabular}{|l|c|}
\hline \multicolumn{1}{|c|}{ Propiedad física } & \multicolumn{1}{|c|}{$\begin{array}{c}\text { Validación } \\
\text { (\%) }\end{array}$} \\
\hline Viscosidad de Casson $(P a . S) h_{C A}$ & 79.0 \\
\hline Esfuerzo mínimo de fluencia de Casson $(\mathrm{Pa}) \tau_{\mathrm{CA}}$ & 86.0 \\
\hline Color $\left(\mathrm{L}^{*}\right)$ & 99.0 \\
\hline Dureza $(\mathrm{N})$ & 98.0 \\
\hline Tamaño de partícula (micras) & 96.0 \\
\hline Promedio & 91.6 \\
\hline
\end{tabular}

\section{Conclusiones y recomendaciones}

Se obtuvo la formulación óptima de chocolate oscuro en tableta al 70 \% de cacao, conformada por la mezcla de 10 partes de CCN 51 con 90 de ICS 6 (pasta de cacao, cocoa en polvo y manteca de cacao) y otros ingredientes: azúcar y lecitina de soya. La optimización aplicando superficie de respuesta, se logró a partir de las propiedades físicas: viscosidad y esfuerzo mínimo de fluencia de Casson, color, dureza, tamaño de partícula, con una validación del 91.6 \%.

Se recomienda utilizar la mezcla de granos de distintos varietales de cacao en la formulación de chocolates para lograr productos de alta calidad, optimizándola aplicando superficie de respuesta.

\section{Bibliografía}

Adeyeye, El., Akinyeye, R. O., Ogunlade, I., Olaofe, O., y Boluwade, J. O. (2010). Effect of farm and industrial processing on the aminoacid profile of cocoa beans. Food Chemistry, 118: 357-363.

Afoakwa, E., Paterson, A., Fowler, M., y Vieira, J. (2008). Particle size distribution and compositional effects on textural properties and appearance of dark chocolates. Journal of Food Engineering, 87 (2): 181-190.

Aidoo, R. P., Afoakwa, E. O., y Dewettink, K. (2014). Optimization of inulin and polydextrose mixtures as sucrose replacers during sugar-free chocolate manufacture-Rheological, microstructure and physical quality characteristics. Journal of Food Engineering, 126: 35-42.

Alvis, A., Pérez, L., y Arrazola, G. (2011). Determinación de las propiedades de textura de tabletas de chocolate mediante técnicas instrumentales. Información tecnológica, 22 (3): 11-18.

AOAC. (2016). Official Methods of Analysis of AOAC International. 18 th Edition, Volume II. Editors: William Horwitz and George W. Latimer, Jr. Maryland, U.S.A.

Bacio, LV. (2007). Optimización multi-objetivo en el problema de metodología de superficie multi-respuesta. Tesis para obtener el grado de maestría en ciencias con especialidad en probabilidad y estadística. Centro de Investigación en matemáticas, A.C., Guanajuato.

Beckett, S. T. (2008). The Science of Chocolate. Cambridge, England: The Royal Society of Chemistry.

Chire, G. C., Valdivia, R. A., Orihuela, C. A. y Ureña, M. O. (2017). Assessment of physical and physicochemical quality of main chocolates traded in Peru. Acta Agronomica, 66 (2): 164-171.

Dwi Saputro, A., Van de Walle, D., Antan Caiquo, B, Hinneh, M., Kluczykoff, M. y Dewettinck, K. (2019). "Rheological behaviour and microstructural properties of dark chocolate produced by combination of a ball mill and a liquefier device as small scale chocolate production system". LWT - Food Science and Technology, 100: 10-19. 
Esan, TA., Sobukola, O. P., Sanni, L. O., Bakare, H. A. y Muñoz, L. (2015). “Process optimization by response surface methodology and quality attributes of vacuum fried yellow-fleshed sweetpotato (Ipomoea batatas L.) chips". Food Bioprod Process, 95: 27-37.

Erdem, O., Gültekin-Özgüven, M., Berktas, I., Ersan, S., Tuna, HE., Karadag, A., Özçelik, B., Günes, G., Cutting, SM. (2014). "Development of a novel synbiotic dark chocolate enriched with Bacillus indicus HU36, maltodextrin and lemon fiber: Optimization by response surface methodology". LWTFood Science and Technology, 56: 187-193.

García, L. (2014). Catálogo de cultivares de cacao del Perú. Lima: Q \& P Impresores S.R.L. Dirección General de Competitividad Agraria. Dirección de Promoción de la Competitividad Dirección de Información Agraria. MINAGRI. Segunda reimpresión.

García, S.M. (2018). "Optimización de la fritura de hojuela de papas nativas (Solanum tuberosum sp.) aplicando el método de superficie de respuesta". Tesis de Maestría, Escuela de posgrado, Universidad Nacional Agraria La Molina, Lima.

Gutiérrez, T.J. (2017). State-of-the-Art Chocolate Manufacture: A Review. Comprehensive Reviews in the Food Science and the Food Safety, 16: 1313-1344.

INACAL. (2017). Norma Técnica Peruana NTP 107.302. Cacao y chocolate. Términos y definiciones. Lima.

INEI. (2018). Instituto Nacional de Estadística e Informática. webinei.inei.gob.pe:8080/SIRTOD1/inicio.html

Kleinert, J. (1980). Tempering of melted chocolate masses. CCB 5, 19-24.

Minifie, B. (1999). Chocolate, cocoa and confectionery. Science and Technology. Third Edition. Maryland, Unite States. Aspen Publishers, Inc.

Miquel, M., Carli, S., Couzens, P. J., Wille, H. J. y Hall, L. D. (2001). Kinetics of the migration of lipids in composite chocolate measured by magnetic resonace imaging. Food Research International, 34: 773-781.

Nelson, RB. y Beckett, ST. 1994. Tuberías, bombas y amasadoras. En: Beckett, ST. (Eds.). Fabricación y utilización industrial del chocolate. Editorial ACRIBIA, S.A. Zaragoza (España).

Ostrowska-Ligeza, E., Marzec, A., Górska, A., Wirkowska-Wojdyła, M., Brys, J., Rejch, A. y Czarkowska, K. (2018). A comparative study of thermal and textural properties of milk, white and dark chocolates. Thermochimica Acta, 1-27. https://doi.org/10.1016/j.tca.2018.11.005

Talancha, O. H. (2014). "Optimización de la incorporación de goma de tara y aceite vegetal en la formulación de una mayonesa ‘light’ para maximizar su aceptabilidad". Tesis de ingeniero, Facultad de Industrias Alimentarias, Universidad Nacional Agraria La Molina, Lima.

Tonfack-Djikeng, F. T., Teyomnou-Teyomnou, W., Tenyang, N., Tiencheu, B., Morfor, AT., Hako-Touko, BA., Ndomou-Houketchang, S., Teboukeu-Boungo, G., Lakshmi-Karuna, MS., Zambou-Ngoufack, F., Macaire-Womeni, H. (2018). "Effect of traditional and oven roasting on the physicochemical properties of fermented cocoa beans". Heliyon, 4, 1-17.

Torres, M. (2012). "Influencia de las características y procesado del grano del cacao (Theobroma cacao) en la composición físico-química y propiedades sensoriales de chocolates". Tesis de doctorado, Escuela Politécnica Superior, Universidad de Vic, Reus.

Tran, P. D; Van Durme, J; Van de Ville, D; De Winne, A; Delbaere, C; De Clercq, N; Pan, T. T. Q; Nguyen, CHP; Tran, D. N. y Dewettinck, K. (2016). Quality attributes of dark chocolate produced from Vietnamese cocoa liquors. Journal of Food Quality 39: 311-322.

Vásquez, O. (2009). Informe Final de Consultoría: "Plan Estratégico de Mercado para la Promoción del Consumo Interno de Derivados del Cacao Nacional". Ministerio de Agricultura. Lima. 1,35.

Windhab, E. J. (2017). Tempering. En Beckett, S. T., Fowler, M. S. y Ziegler, G. R. (eds.). Beckett`́s Industrial Chocolate Manufacture and Use. Fifth Edition. York, United Kingdom. Wiley-Blackwell.

Wohlmuth, EG. (2017). Recipes. In: Beckett, S. T., Fowler, M. S. y Ziegler, GR. (eds.). Beckett’s Industrial Chocolate Manufacture and Use. 5. ${ }^{a}$ ed.. York, United Kingdom: Wiley-Blackwell.

Wolf, B. (2017). Chocolate Flow Properties. En Beckett, S. T., Fowler, MS. y Ziegler, G. R. (eds.). Beckett’s

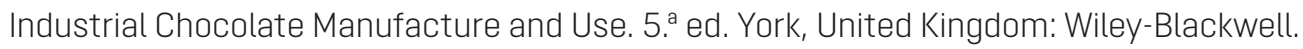

